\title{
A PREDICTION METHOD OF GESTURE TRAJECTORY BASED ON LEAST SQUARES FITTING MODEL
}

\author{
Cai Mengmeng ${ }^{1,2}$ Feng Zhiquan ${ }^{1,2}$ and Luan Min ${ }^{1,2}$ \\ ${ }^{1}$ School of Information Science and Engineering, \\ University of Jinan, Jinan, China 250022 \\ ${ }^{2}$ Shandong Provincial Key Laboratory of Network-based Intelligent Computing, \\ Jinan, China, 250022, P.R.
}

\begin{abstract}
Implicit interaction based on context information is widely used and studied in the virtual scene. In context based human computer interaction, the meaning of action A is well defined. For instance, the right wave is defined turning paper or PPT in context B. And it mean volume up in context $C$. However, we cannot use the context information when we select the object to be manipulated. In view of this situation, this paper proposes using the least squares fitting curve beam to predict the user's trajectory, so as to determine what object the user's wants to operate. At the same time, the fitting effects of the three curves were compared, and the curve which is more accord with the hand movement trajectory is obtained. In addition, using the bounding box size control the $Z$ variable to move in an appropriate location. Experimental results show that the proposed in this paper based on bounding box size to control the $Z$ variables get a good effect; by fitting the trajectory of a human hand, to predict the object that the subjects would like to operate. The correct rate is $91 \%$.
\end{abstract}

\section{KEYWORDS}

Least-squares method; dynamic gesture recognition; implicit interaction; edge point sequential extraction; context information

\section{INTRODUCTION}

With the continuous development of computer science and technology, intelligent humancomputer interaction has gradually become the dominant trend in the development of computing model. And this trend becomes more obviously after Weiser Mark [1] putting forward the concept of "Ubicomp" in 1990s. In order to lighten the load of people's operation and memory, during the interaction, the traditional way of interaction need to be expanded. And integrate the implicit human-computer interaction into the explicit human-computer interaction.

At present, implicit human-computer interaction has become an important research frontier in the field of interaction. The universities and research institutes of the United States, Germany, China, Austria and so on, has been carried out in-depth study to IHCI theory and application gradually. Schmidt in the University of Karlsruhe in Germany conducted an earlier study of the theory of

DOI : $10.5121 /$ vlsic. 2017.8101 
implicit interaction [2].He believes that the two elements of implicit interaction are perception and reasoning, and he also put forward that contextual information is very important for interaction. Young-Min Jang [3] proposed a novel approach for a human's implicit intention recognition based on the eyeball movement pattern and pupil size variation. Hamid Mcheick [4] presents a context aware model with ability to interact. This model adapts to dynamically environment and can interact with the user flexibility. The implicit interaction based on context is also applied in other aspects. $\mathrm{R}$ Cowie [5] proposed an emotion-recognising system to recognize emotion. Bojan Blažica [6] introduces a new more personal perspective on photowork that aims at understanding the user and his/her subjective relationship to the photo. It does so by means of implicit human-computer interaction, this is, by observing the user's interaction with the photos.

In China, Tao Linmi [7] of Tsinghua University developed an adaptive vision system to detect and understand user behaviour, and to carry out implicit interaction. At the same time, Tian Feng in software research institute of Chinese Academy of Sciences also studied the characteristics of implicit interaction from the perspective of post WIMP user interface [8]. Wang Wei proposes that more use of user context information in the process of implicit humancomputer interaction [9], including user behaviour, emotional state (for example: the emotional design method of Irina CRISTESCU[10] ), and physiological state. But there is also some use of environmental context information, such as location-based services, etc. And it pointed out that the implicit humancomputer interaction technology is one of the development directions in the future. Feng Zhiquan [11] uses the context information in the gesture tracking, and has achieved a good interaction effect. Song Wei [12] uses multi-modal input and context-aware computing to combine gesture recognition into the game's scene control. This method allows users to feel the reality of people and the role of the game the perfect fusion. Yue Weining [13] proposed a context aware and scheduling strategy for intelligent interactive systems, which improves the system's intelligence.

\section{RELATED WORK}

\subsection{Image segmentation}

Before image segmentation, the image should be filtered to remove the noise. At present, the common methods of image segmentation [14] can be divided into: threshold segmentation method [15], edge detection method [16], region segmentation method and the method of combining the theorem of the segmentation method. Besides, Qu Jingjing[17] proposed the segmentation method of continuous frame difference and background subtraction. This article uses the skin colour model [15] (YcbCr) to separate the human hand and the background, and the image banalization. The formula for converting from RGB colour-space to $\mathrm{YCbCr}$ colourspace is shown in formula 1 .

$$
\left[\begin{array}{l}
Y \\
C b \\
C r
\end{array}\right]=\left[\begin{array}{ccc}
0.2990 & 0.5870 & 0.1140 \\
-0.1686 & -0.3313 & 0.5000 \\
0.500 & -0.4187 & -0.0813
\end{array}\right]\left[\begin{array}{l}
R \\
G \\
B
\end{array}\right]+\left[\begin{array}{c}
0 \\
128 \\
128
\end{array}\right] \text { (formula 1) }
$$

Segmentation results are shown in fig 1: 

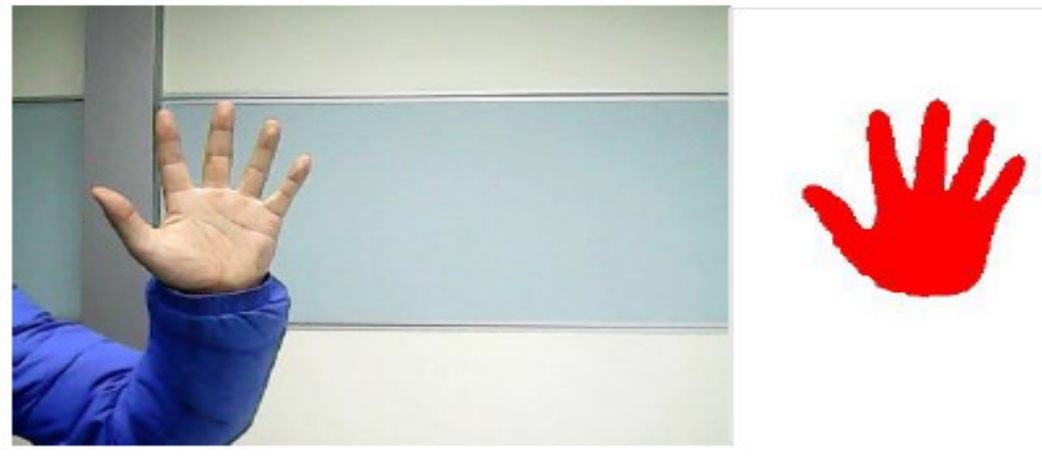

Figure 1. Original image and segmented image

\subsection{Feature Extraction}

According to the formula (2) get the centroid coordinates of mass [18] as shown in fig. 2 the green point inside the palm.

$$
\mathrm{r}_{c}=\frac{\sum_{i} m_{i} r_{i}}{\sum_{i} m_{i}}
$$

The eight-neighbourhood tracking algorithm [19] is used to counter clockwise extract contour points. In the obtained contour points, according to the formula 3, calculated the three corners' points (we define the points in the profile where the gradient is abrupt as key points, such as the fingertip and the finger-root) closest to the centroid.

$$
\left.H_{i}=\sqrt{\left(x_{i}-x_{c}\right)^{2}+\left(y_{i}-y_{c}\right)^{2}} \quad \text { (formula } 3\right)
$$

As show in fig 2, the green points are feature points.
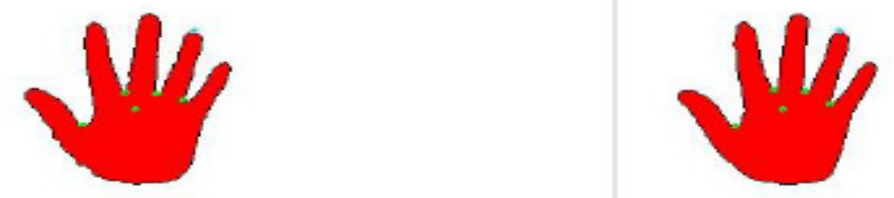

Figure 2. Feature points extraction

\subsection{Dynamic gesture recognition}

In his paper [20], he proposed the method that according to the change in the angle between the negative $\mathrm{x}$-axis and the straight line of the centroid and middle finger to recognize the clockwise or counter clockwise rotation. And according to the finger-tip and finger-root position changes (mainly include the distance changes of finger-tip and adjacent finger-root, the distance changes 
of finger-tip and adjacent finger-tip, the same finger-tip and finger-root between different frames), to identify the moving, grasping and putting. According to the direction of hand movement to identify move up, down left and right. In this paper, this approach is used for dynamic gesture recognition.

\section{SCENE MODELLING}

\subsection{Brief introduction of image display}

The principle of image display using OpenGL [21] in the virtual environment is exemplified here in fig 3 .

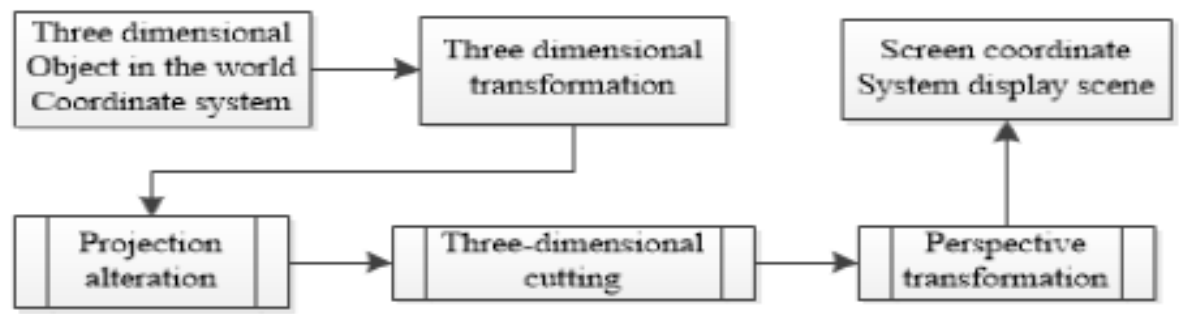

Figure 3. The principle of OpenGL image display

For different $\mathrm{Z}$ plane $(\mathrm{Z}=\mathrm{C}, \mathrm{C}$ is a constant $)$, moving the same distance in one condition while the output of distance is not the same (the closer to the point of view, the greater the moving distance on the screen is). Therefore, objects at different coordinates in the virtual scene needs different functions to move them. Moreover, two-dimensional image obtained by the common camera is not good at controlling the movement of three - dimensional hand in three - dimensional spaces. So many researchers have used animation as a method to avoiding this problem. Using the principle that the bounding box size is proportional to the image display is the key to control changes in the $\mathrm{Z}$-axis coordinate.

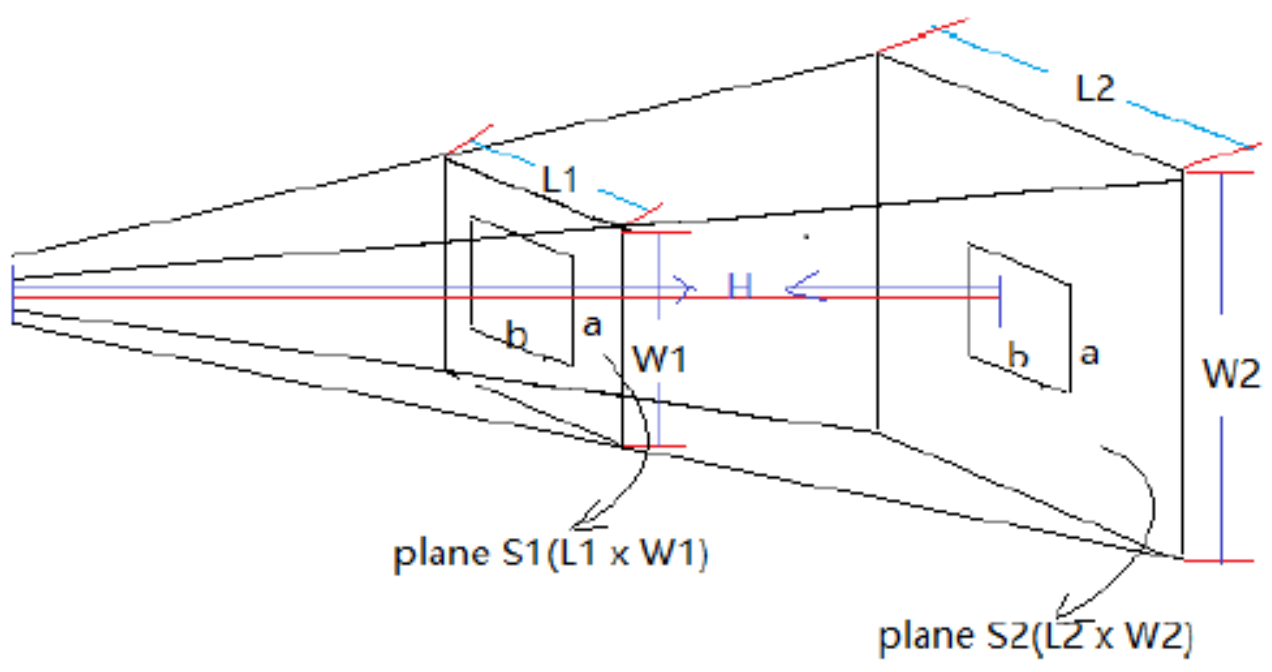

Figure 4. Camera image acquisition principle 
International Journal of VLSI design \& Communication Systems (VLSICS) Vol.8, No.1, February 2017

The captured image (the size of determined acquisition: 400x300) is mapped to "a" window to display. So the length of "a" in plane S1 is $\frac{W_{2}}{W_{1}}$ times in plane $S 2$ at the time of display.

\subsection{Determine the relationship of mapping}

Collect and record the size of the bounding box and get its average size when each of the experimenter is operating in the 3D scene. And mapping shown by MATLAB is shown in fig 5.
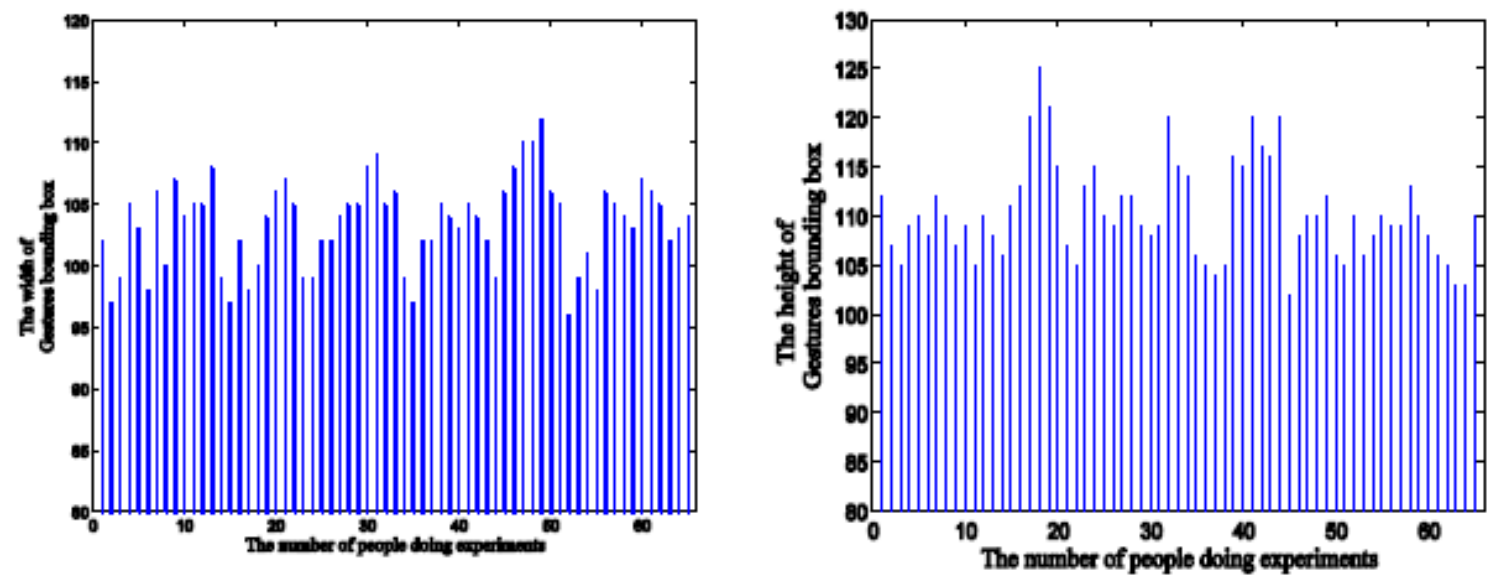

Figure 5. The height and width of gesture bounding box

According to the probability formula in statistics:

$$
\begin{aligned}
& L=\frac{1}{n} \sum_{i=0}^{n-1} 1_{i} \\
& W=\frac{1}{n} \sum_{i=0}^{n-1} W_{i}
\end{aligned}
$$

(formula 5)

Calculate the initial value of $\mathrm{L}$ and $\mathrm{W}: \mathrm{L}_{0}=110, \mathrm{~W}_{0}=80$

Calculate the corresponding relationship between real and virtual human hand (when the bounding box keeps the same size).

$$
\begin{aligned}
& \frac{\Delta L}{\Delta \dot{L}^{\prime}}=\frac{400-2 * L_{0}}{\dot{L}_{0}}=0.18125 \\
& \frac{\Delta W}{\Delta W^{\prime}}=\frac{300-2 * W_{0}}{W_{0}^{\prime}}=0.1833
\end{aligned}
$$

(formula 6)

(formula 7)

In the virtual scene, $\mathrm{L}_{0}^{*}=1600, \mathrm{~W}_{0}^{\prime}=1200$ - Real hand moves in the horizontal direction a unit, virtual hands should move 5.51 units; and moving a unit in the vertical direction means virtual 
hands should move 5.45 units. For other positions virtual hands should move $5.51 \frac{\mathrm{L}}{L_{\mathrm{n}}}$ units; virtual hands should move $5.45 \frac{\mathrm{W}}{W_{0}}$ units.

For $\mathrm{Z}$ coordinates, Position of each object in virtual scene is in [20 30]. The variation of bounding box's length is 80 pixels to 130 pixels. So the congruent relationship of bounding box's length and $\mathrm{Z}$ coordinates is:

$$
\frac{Z-20}{L-80}=\frac{30-20}{130-80}=0.2 \quad \text { (formula 8) }
$$

That is :

$$
\mathrm{z}=20+(1-80) * 0.2
$$

\section{INTERACTION ALGORITHM BASED ON SCENE SITUATION AWARENESS}

\subsection{Based on least square method [22] to fit the motion trajectory algorithm}

The movement trajectory of human-hand in the virtual scene is the circular motion to the elbow joint as the centres of the arc. So in order to better fit the motion trajectory of the hand gesture, in this paper, the least square method is used to fit the nonlinear equation.as shown in formula 9-1. At the same time, this paper also uses linear (formula 9-2) and parabolic (formula 9-3) trajectories. Take the first curve (formula 9-1) as an example to introduce the least square method

$$
\begin{array}{rlrl}
\mathrm{y}_{i}=a * x_{i}+b * \sin \left(x_{i}\right)+c & (\mathrm{i}=1,2,3, \ldots, \mathrm{n}) & (\text { formula } 9-1) \\
\mathrm{y}_{i}=a * x_{i}+\mathrm{c} & (\mathrm{i}=1,2,3, \ldots, \mathrm{n}) & & (\text { formula } 9-2) \\
\mathrm{y}_{i}=a * x_{i}{ }^{2}+b * x_{i}+c & (\mathrm{i}=1,2,3, \ldots, n) & & (\text { formula } 9-3)
\end{array}
$$

In formula 9, the point $\left(x_{i}, y_{i}\right)$ is the observation coordinate, "a" is first-order coefficients, " $b$ " is sine coefficients, and $\mathrm{c}$ is constant. "a", " $\mathrm{b}$ " and $\mathrm{c}$ is the parameter to be solved, assume $\mathrm{a}_{0}, \mathrm{~b}_{0}, \mathrm{c}_{0}$ for their approximate value.

Order:

$$
\begin{aligned}
& \mathrm{a}=a_{0}+\delta a, \\
& \mathrm{~b}=b_{0}+\delta b, \\
& \mathrm{c}=\mathrm{c}_{0}+\delta c
\end{aligned}
$$

Taking $\mathrm{y}$ as the dependent variable and $\mathrm{x}$ as the independent variable, the error equation is: 
International Journal of VLSI design \& Communication Systems (VLSICS) Vol.8, No.1, February 2017

$$
\mathrm{v}_{y i}=\left[\begin{array}{lll}
x_{i} & \sin \left(x_{i}\right) & 1
\end{array}\right]\left[\begin{array}{l}
\delta \mathrm{a} \\
\delta b \\
\delta c
\end{array}\right]+a_{0} * x_{i}+b_{0} * \sin \left(x_{i}\right)+c_{0}-y_{i} \text { (formula 10) }
$$

Error equation matrix can be expressed as:

$$
A \delta X=1+V \quad \text { (formula 11) }
$$

Among them:

$$
\begin{aligned}
& A=\left[\begin{array}{ccc}
x_{1} & \sin \left(x_{1}\right) & 1 \\
x_{2} & \sin \left(x_{2}\right) & 1 \\
\vdots & \vdots & \vdots \\
x_{2} & \sin \left(x_{n}\right) & 1
\end{array}\right], \delta X=\left[\begin{array}{c}
\delta a \\
\delta b
\end{array}\right] \\
& I=\left[\begin{array}{c}
a_{0} x_{1}+b_{0} \sin \left(x_{1}\right)+c_{0}-y_{1} \\
a_{0} x_{2}+b_{0} \sin \left(x_{2}\right)+c_{0}-y_{2} \\
\vdots \\
a_{0} x_{n}+b_{0} \sin \left(x_{n}\right)+c_{0}-y_{n}
\end{array}\right], V=\left[\begin{array}{c}
V_{y 1} \\
V_{y 2} \\
\vdots \\
V_{y n}
\end{array}\right]
\end{aligned}
$$

According to the least square rule (formula 12), fitting a curve.

$$
\sum_{i=1}^{n}\left\|a x_{i}-b \sin \left(x_{i}\right)+c_{0}-y_{i}\right\|^{2}=\min \quad \text { (formula 12) }
$$

And dependent variable residual is:

$$
\mathrm{V}=A \delta X-1
$$

(formula 13)

Because the cycle of $\sin \left(x_{i}\right)$ is $2 * \pi, \mathrm{b} \sin \left(x_{i}\right)$ is periodic oscillation among in [0 400], so equation of a curve is

$$
\mathrm{y}_{i}=a x_{i}+b \sin \left(0.01 x_{i}\right)+c \quad(i=1,2, \ldots, n)
$$

In the end, according to the coefficient to confirm the fit is good or bad.

\subsection{Scene situation awareness and interaction algorithm}

Calculate the size of the bounding box, and determine the corresponding relationship. According to the moving direction and distance of the 3D human hand of the two frame image, the movement of the centroid of the human hand is determined. The feature data of the multi frame images is used to synthesize nonlinear curve to predict the direction of human hand movement. 
International Journal of VLSI design \& Communication Systems (VLSICS) Vol.8, No.1, February 2017

And then determine the object at the direction and get the distance to human hand. Therefore, perform the corresponding operation; the specific algorithm is as follows:

First step: Capture a RGB image using a common camera .The height of the image is 400, and the width is 300. Then carry out image segmentation using skin colour model, and image banalization.

Second step: Calculate the centroid coordinates, and the coordinates of the three corner points. According to the formula 14 figure out bounding-box size.

$$
\begin{aligned}
& \mathrm{X}_{1}=\min _{f\left(x_{i}, y_{i}\right) \rightarrow 0}\left\{X_{i}\right\} \\
& \mathrm{X}_{\mathrm{r}}=\max _{f\left(x_{i}, Y_{i}\right) \sim 0}\left\{X_{i}\right\} \quad \text { (formula } 14-2 \text { ) } \\
& \mathrm{Y}_{l}=\min _{f\left(x_{i}, y_{i}\right)=0}\left\{\mathrm{y}_{i}\right\} \quad \text { (formula } 14-3 \text { ) } \\
& \left.\mathrm{Y}_{r}=\max _{f\left(x_{i}, y_{i}\right) \rightarrow 0}\left\{\mathrm{y}_{i}\right\} \quad \text { (formula } 14-4\right)
\end{aligned}
$$

Among of them, the $X_{1}$ is the left edge of the bounding box, the $X_{r}$ is right edge; the $Y_{I}$ is the upper boundary of the bounding box, and the $Y_{r}$ is lower boundary. The equation $\mathrm{f}\left(x_{i}, y_{i}\right) \neq 0$ means that the pixels of the $\left(\mathrm{x}_{i}, y_{i}\right)$ coordinates are skin colour.

Third step: Calculate the change between the two images, include centre of mass and corner point, According to the size and coordinates of the bounding box, and determine the direction and distance of the hand's move in the 3D virtual scene.

$$
(\delta \mathrm{x}, \delta y)=\left(x_{i+1}, y_{i+1}\right)-\left(x_{i}, y_{i}\right)=\left(x_{i+1}-x_{i}, y_{i+1}-y_{i}\right)
$$

Fourth step: Using the glTranslatef (Dx, Dy, Dz) belonging to OpenGL to change the movement of the three-dimensional human hand in the virtual environment. If the moving amount of one direction (assumed to be $\mathrm{X}$ axis direction) is much greater than the other direction ( $\mathrm{Y}$ axis) so you can only consider the direction where the moving amount is larger.

Fifth step: Judge whether frames is greater than a threshold (set to 10). If less than, then return to the first step; Else, Using the least squares method to fit the data stored characteristic.

Sixth step: According to the correlation coefficient, judge whether the curve fitted is good or not. If good, go to step seven; if not, find the number of the points that cause poor fitting, If only one or two, then discard the two data and frames minus two, return to the fourth step. If more throw away the data before that location and frames minus corresponding to the value, return to the fourth step.

Seventh step: Determine the number of objects that are in the prediction direction; if not one, frames minus five, and return to the fourth step. If there is only one: moving the corresponding object to the virtual human hand with the same speed, and judges the distance between the virtual 
International Journal of VLSI design \& Communication Systems (VLSICS) Vol.8, No.1, February 2017

human hand and the object. If the distance is less than the threshold, stop moving the object, and go to the last step.

At last, carry out the corresponding operation on the object by identifying a series of actions, for example: rotation, scaling, translation, and so on.

Algorithm flow chart is shown in fig 6.

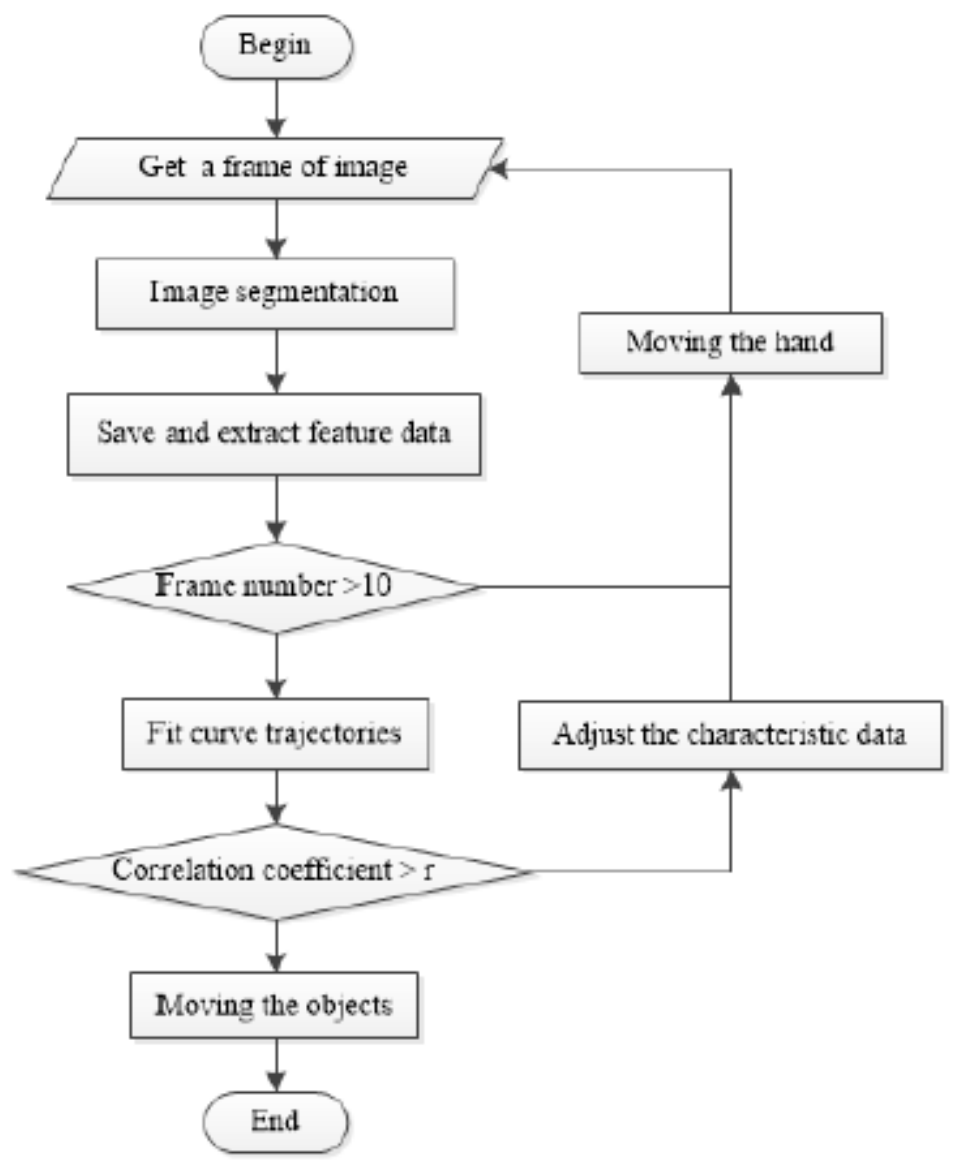

Figure 6. Algorithm flow chart

\section{EXPERIMENTAL RESULT}

The experiment is divided into three parts. One part is to be familiar with the experimental environment, operation method and procedure, to determine the mapping relationship. Experimental environment and virtual interface are shown in fig 7.

On the right is a virtual 3D scene. Scene consists of virtual hands, and desktop, there is a cube, small ball, cylinder and teapot on the table. Each object is fixed, and is not in a z plane. On the left, there have two pictures, one picture is the original, and the other is the split hand. The change of real hand and virtual hand has a certain relationship. And this relationship is determined by the distance of the real hand from the camera. 
International Journal of VLSI design \& Communication Systems (VLSICS) Vol.8, No.1, February 2017

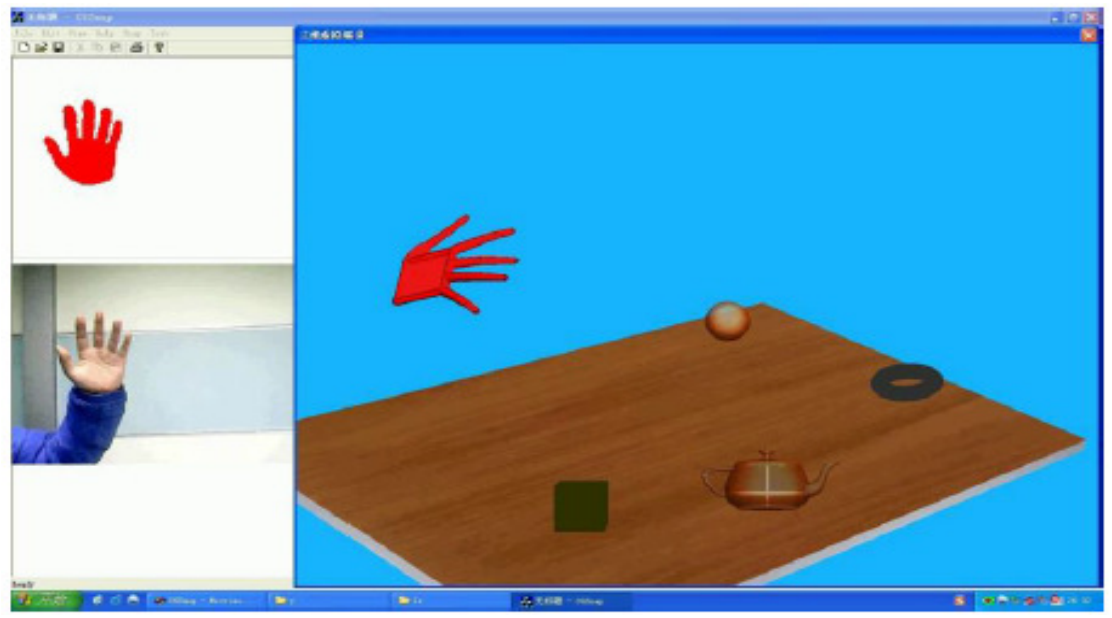

Figure 7. Virtual scene

I find 65 students to do the experiment in the laboratory environment, under the constant light environment, the completion of the virtual scene move towards the ball, the cube, the cylinder, and the teapot in the experimental environment. I recorded the size of their hand gestures when they were in the experiment, calculate the average and mapped with MATLAB, as shown in the fig 5. Determine the corresponding relationship and discrete table data, the content of the discrete table is related to the size of the bounding box and the speed of motion.

The second part is verifying this method which through the hand's trajectory to predict the object you want to operate on is correct. Look for 100 experimenters; let them familiar with the operation of the experimental environment. When they can skilfully move the object they want to operate, start the experiment and collect the data. Tell them to move their hands control the virtual hand towards the ball, the cylinder, and the teapot in the experimental environment. Record their manual centre's trajectory of mass until collision detection is complete. And output the data to a text file. Experimental data is divided into two, one is to contain the movement data of collision detection, and the other does not have. I import the experimental data into MATLAB, use the following code to fit the dashed line.

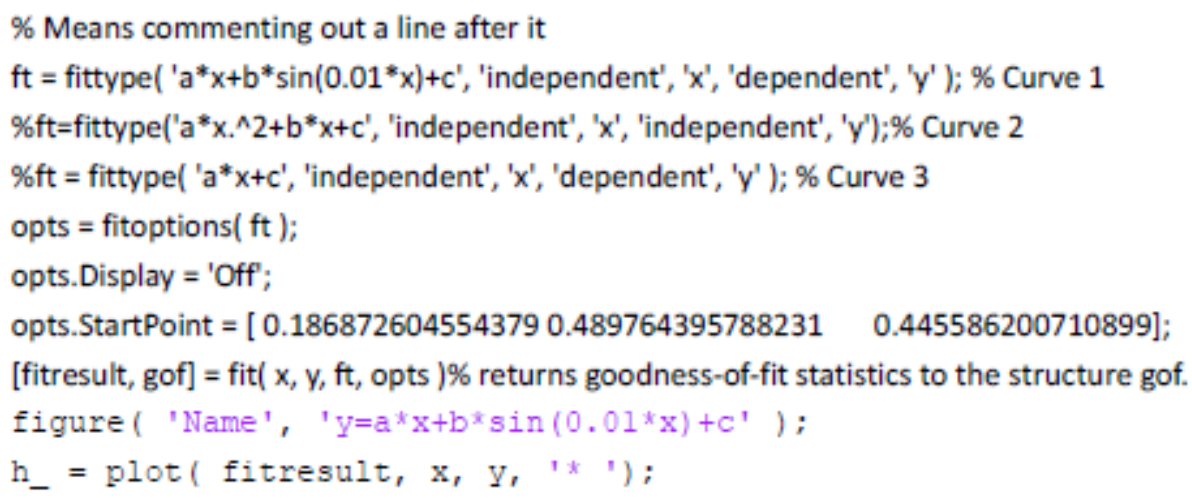


International Journal of VLSI design \& Communication Systems (VLSICS) Vol.8, No.1, February 2017

In the code, the gof structure has the fields shown in the table below. And the three curves were used to fit these data.

Table 1. Parameter of gof

\begin{tabular}{|l|l|}
\hline Field & Value \\
\hline sse & Sum of squares due to error \\
\hline rsquare & Coefficient of determination \\
\hline dfe & Degrees of freedom \\
\hline adjsquare & Degree-of-freedom adjusted coefficient of determination \\
\hline rmse & Root mean squared error(standard error) \\
\hline
\end{tabular}

According to the experimental data of move to the ball to fit curve, as shown in the fig 8 .
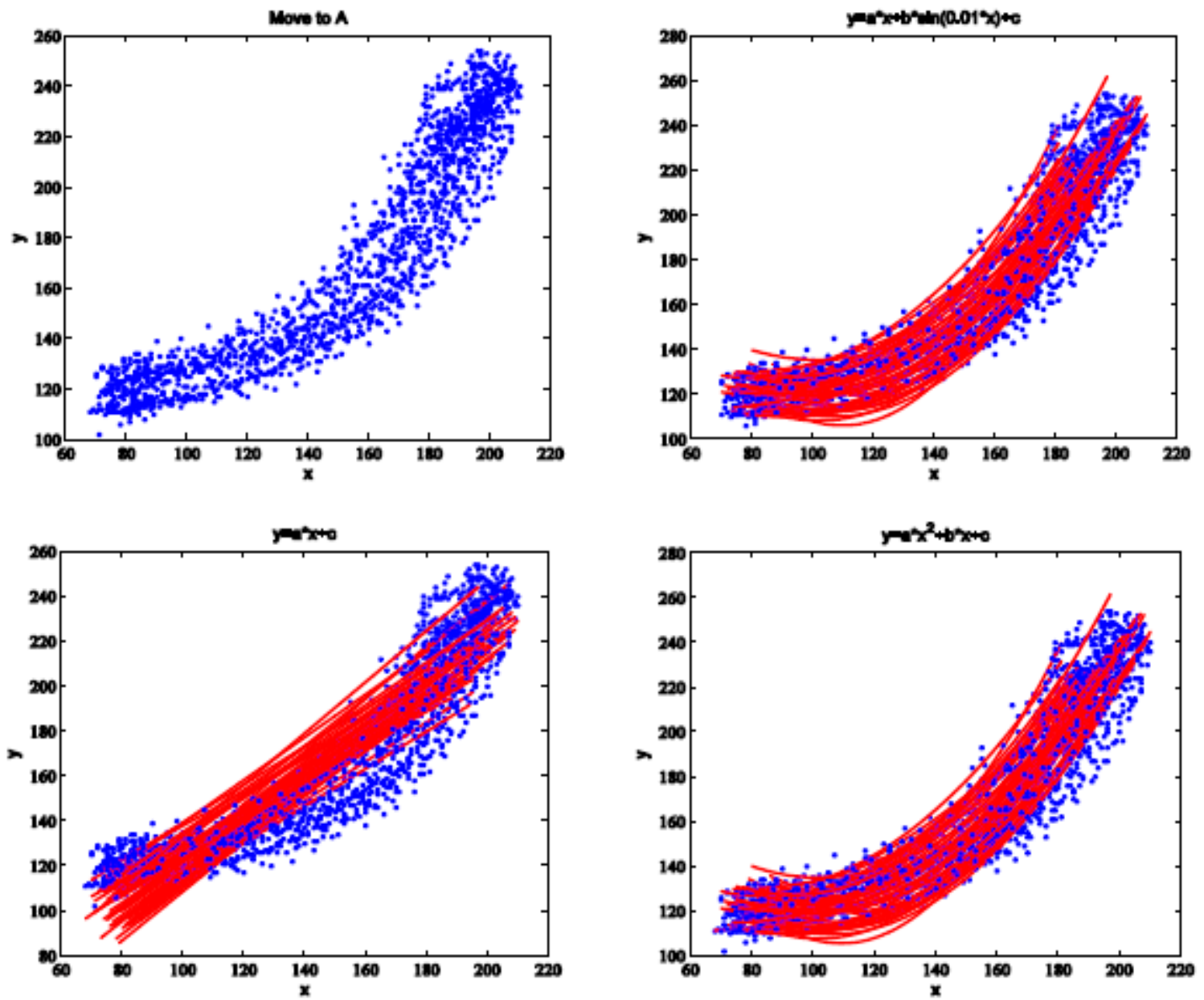

Figure 8. Matlab fitting curve

In the fig 8, the horizontal axis is the abscissa of the picture where the pixel points, the vertical axis are the ordinate of the picture where the pixel points, the blue point are the trajectory of the human hand. The red line is the fitted curve. From these four figures we can see that the trajectory of the human hand can be predicted by fitting the curve. The following is a fitting image (as show 
International Journal of VLSI design \& Communication Systems (VLSICS) Vol.8, No.1, February 2017

in fig 9) from the same one of the experimental. On the left is a fitting curve with collision detection, on the right side is the fitting curve of the same function without collision detection. As can be seen from the image, the curve on the left and right sides of the changes have very little difference; you can even use less data fitting similar curves. However, the fewer points are fitted, the less time is spent. Of course, the completion of an experimental points and the speed of movement is related.
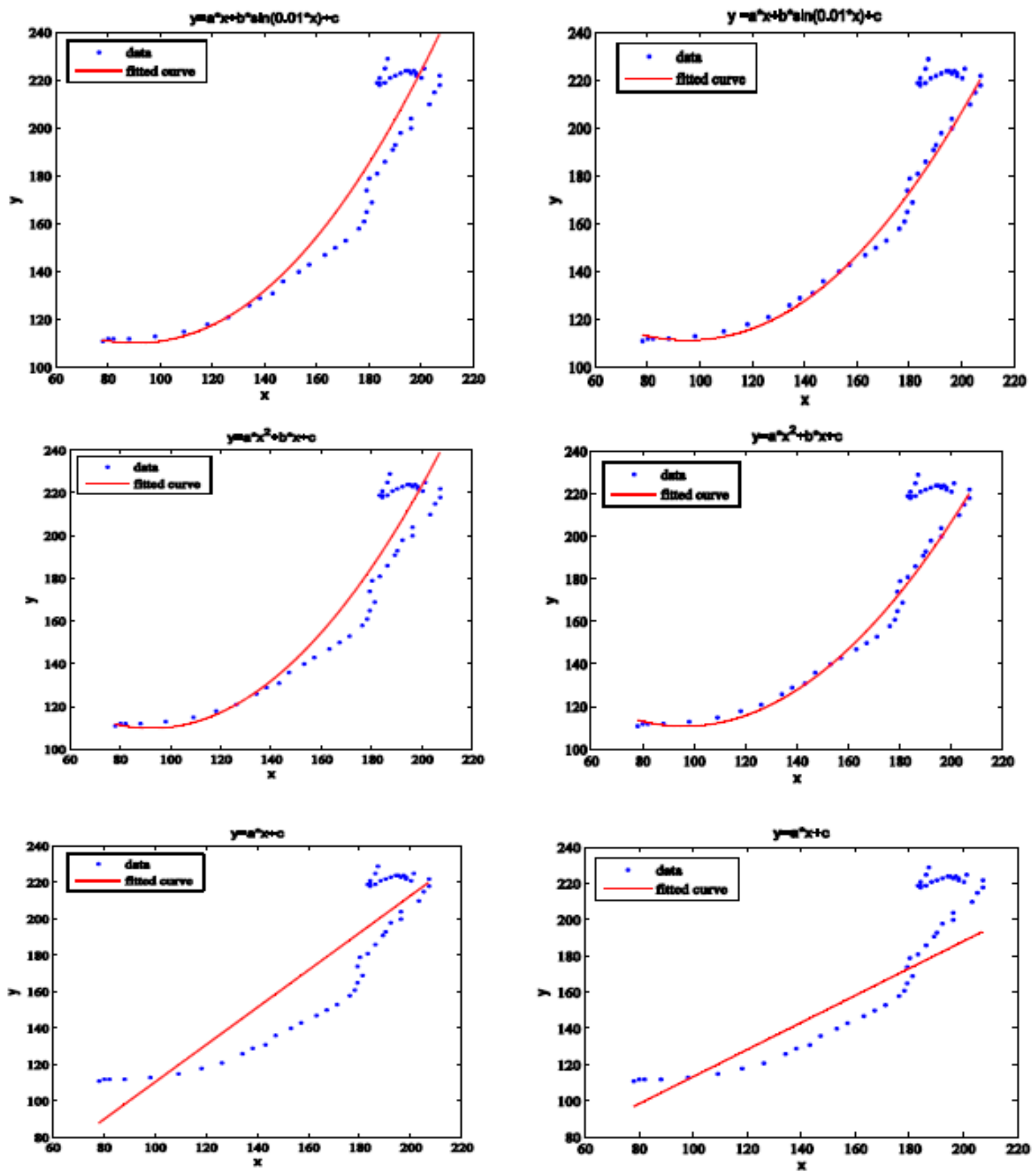

Figure 9. Fitting graphs of three curves 
International Journal of VLSI design \& Communication Systems (VLSICS) Vol.8, No.1, February 2017

In addition, I also obtained six parameters (gof) of three fitting curves as shown in fig 10.

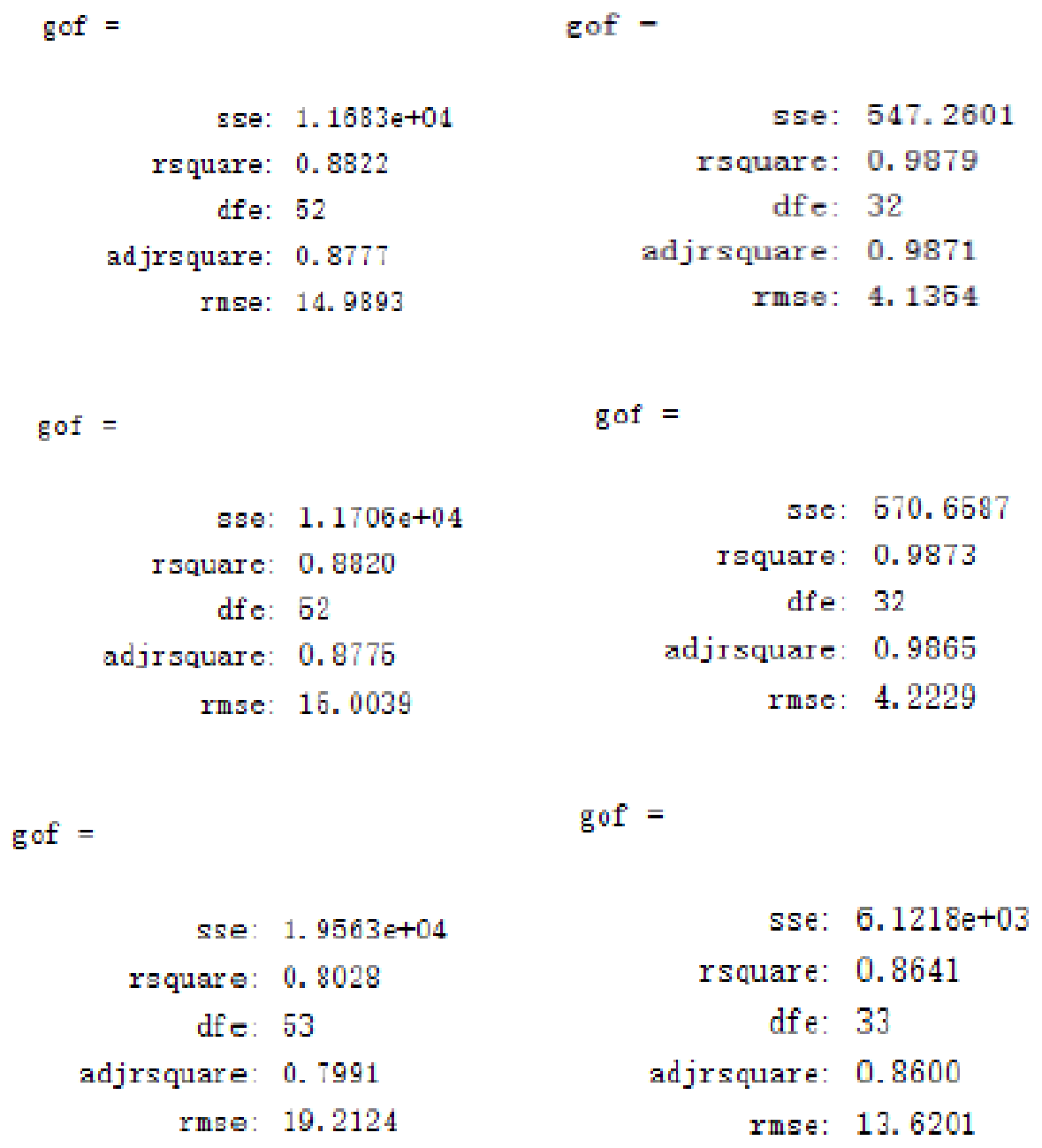

Figure 10. Parameters of three fitting curves

As can be seen from the fig 10, the straight line fitting effect is the worst; the correlation coefficient is only $0.79,0.86$. And the correlation coefficients of the other two fitting methods are similar. Get all the experimental curve fitting correlation coefficient, and calculate their average of three fitting curves. As shown in fig 11 


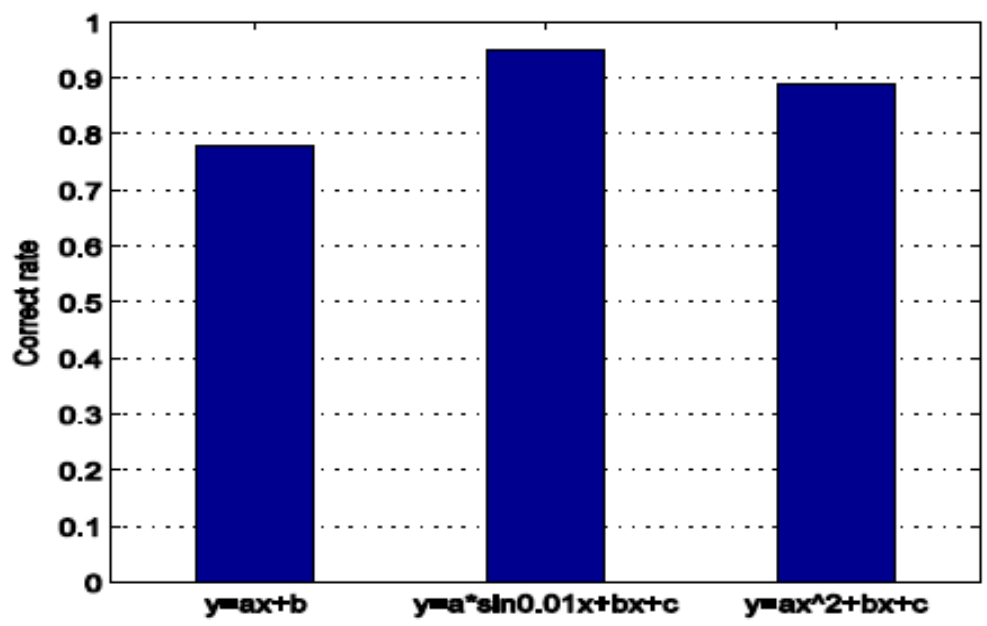

Figure 11. Average correlation coefficient

From the figure we can see, Curve $\mathrm{y}=\mathrm{a} * \mathrm{x}+\mathrm{b} * \sin (0.01 * \mathrm{x})+\mathrm{c}$ has the best fitting effect. And the sin function is a function describing the circular motion. It fits the movement of hand.

The last part is verifying the correctness of the forecast results in this method. Look for 100 experimenters again, let them familiar with the operation of the experimental environment. Tell them to move their hands control the virtual hand towards the ball, the cylinder, and the teapot in the experimental environment. And the speed of hand movement is moderate. When they can skilfully move the object they want to operate, start the experiment at different distances and collect the data. Record the number of frames for each person to complete the experiment. In the experimental process with predictive function, according to the predicted result, it is judged which object in the virtual scene is to be selected. It will move the object to the virtual hand and save the current number of frames. Wait the end of the experiment, record whether the forecast is correct or not. Repeat the experiment 5 times per person, seek its average. The final results are shown in Table 2.

Table 2. Experimental prediction results

\begin{tabular}{|c|c|c|c|c|c|}
\hline Correct Predicted Times & 1 & 2 & 3 & 4 & 5 \\
\hline The number of people & 0 & 0 & 8 & 29 & 63 \\
\hline
\end{tabular}

Through the table 2 we can be seen most people can predict success 5 times, and a little of people predicted success 3 times, nobody can predict success less than 3 times. So, we can get a conclusion: in a specific virtual environment, the use of curve fitting method can be very good to predict the subjects want to operate the object. The 8 picture in fig 10 is the screen in the experimental process with predictive function.

In the fig 12 the real hand controls virtual hand to move the ball, and the system predicts the moving direction of the virtual hand based on the motion data. Then the object in this direction is moved to the virtual hand, as you can see in the last two pictures the red balls move toward the virtual hand. This shows the prediction is correct 


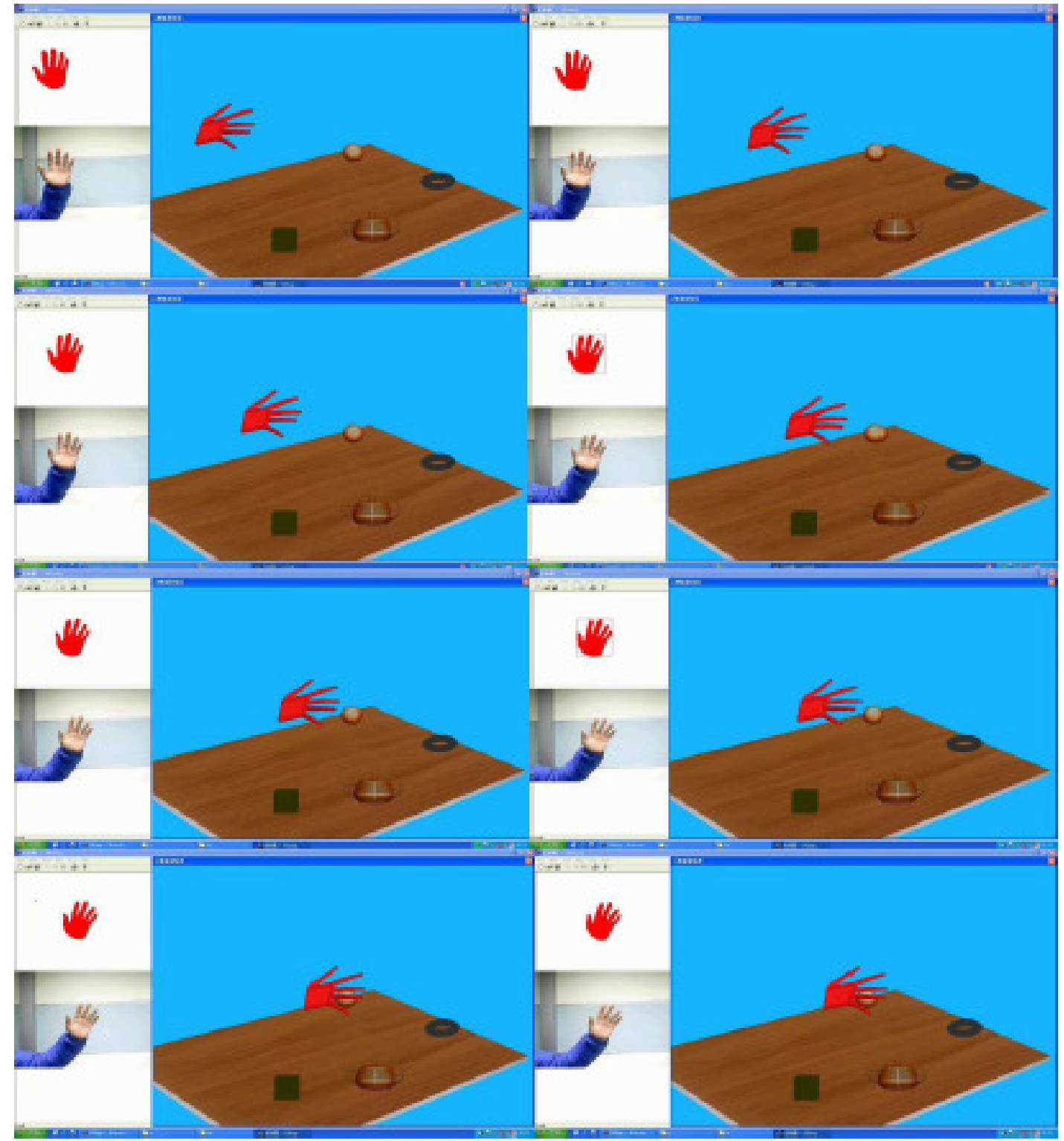

Figure 12. Move to the red ball

\section{CONCLuSiOnS}

In this paper, according to the characteristics of human motion in the virtual scene, we propose to fit the motion trajectory according to the change of human hand's centroid, and then to predict the objects that people want to operate. According to the characteristics of trajectory points, three common curves are used to fit the same trajectory. By comparing the fitting parameters, it is finally decided to use curve $\mathrm{y}=\mathrm{a} * \mathrm{x}+\mathrm{b} * \sin (0.01 * \mathrm{x})+\mathrm{c}$ to fit the trajectory. A large number of experiments show that this method can be a good predictor of the experiment want to operate the object. And it can shorten the time of selecting an object. In addition through the bounding box size changes, to control the virtual object $\mathrm{Z}$ coordinate changes in the appropriate range. It conforms to the people in the three-dimensional environment in the operation of the habit (hand 
International Journal of VLSI design \& Communication Systems (VLSICS) Vol.8, No.1, February 2017

before and after the change, the virtual hand before and after the move). It also achieved good interaction effects. But for implementation a more intelligent human-computer interaction, there are a lot of problems to be solved. For example: the occlusion problem, as well as the computer automatically judge whether people have the purpose of the operation, etc.

\section{ACKNOWLEDGEMENTS}

This work is supported by National Natural Science Foundation of China under Grant No. 61472163, partially supported by the National Key Research I\& Development Plan of China under Grant No.2016YFB1001403, Science and technology project of Shandong Province under Grant No. 2015GGX101025

\section{REFERENCES}

[1] Weiser Mark . The computers for the twenty-first century [J].Scientific American, 1991, 265(3) : 94104

[2] Schmidt A. Implicit human computer interaction through context [J] .Personal Technologies, 200, 4 (2/3):191-199.

[3] Young-Min Jang, Rammohan Mallipeddi, Sangil Lee, Ho-Wan Kwak, Minho Lee. Human intention recognition based on eyeball movement pattern and pupil size variation [J]. Neurocomputing, 2013.

[4] Hamid Mcheick. Modeling Context Aware Features for Pervasive Computing [J]. Procedia Computer Science, 2014, 37.

[5] Cowie R, Douglascowie E, Tsapatsoulis N, et al. Emotion recognition in human-computer interaction[J]. Neural Networks the Official Journal of the International Neural Network Society, 2001, 18(1):32-80.

[6] Bojan Blažica, Daniel Vladušič, Dunja Mladenić. A personal perspective on photowork: implicit human-computer interaction for photo collection management [J]. Personal and Ubiquitous Computing, 2013, 178

[7] Wang Guojian, Tao Linmi. Distributed Vision System for Implicit Human Computer Interaction [J]. Journal of Image and Graphics, 2010,08:1133-1138

[8] TIAN Feng, DENG Changzhi, ZHOU Mingjun, et al. Research on the implicit interaction characteristic of Post-WIMP user interface. Journal of Frontiers of Computer Science and Technology, 2007, 1(2): 160- 169.

[9] WANG Wei, HUANG Xiaodan, ZHAO Jijun, et al. Implicit Human_Computer Interaction [J]. Information and Control, 2014, 01:101-109.

[10] Irina CRISTESCU. Emotions in human-computer interaction: the role of nonverbal behaviour in interactive systems [J]. Informatica Economica Journal, 2008, XII2:.

[11] Feng ZQ, Yang B, Zheng YW, Xu T, Tang HK. Hand tracking based on behavioural analysis for users. Ruan Jian Xue Bao/Journal of Software, 2013,24(9):2101-2116 (in Chinese). http://www.jos.org.cn/1000-9825/4368.htm 
International Journal of VLSI design \& Communication Systems (VLSICS) Vol.8, No.1, February 2017

[12] Song Wei. Research and Implementation of An Online Game Based on Context Information of Mobile Phone[D]. Beijing University of Posts and Telecommunications, 2011

[13] Yue Weining, Wang Yue, Wang Guoping et al. Architecture of Intelligent Interaction Systems Based on Context Awareness [J]. Journal of computer-Aided Design and Computer Graphics, 2005, 01:7479 .

[14] S. M. Lock, D. P. M. Wills. VoxColliDe: Voxel collision detection for virtual environments[J]. Virtual Reality, 2000, 51: .

[15] Haokui-tang. Study of Skin Segmentation Based on Double-Models [D]. Shandong University, 2009.

[16] Lu Kai, Li Xiaojian, Zhou Jinxing. Hand Signal Recognition Based on Skin Colour and Edge Outline Examination [J]. Journal of North China University of Technology, 2006, 03:12-15.

[17] QU Jing-jing, XIN Yun-hong. Combined Continuous Frame Difference with Background Difference Method for Moving Object Detection [J]. Acta Photonica Sinica, 2014, 07: 219-226.

[18] Zhang Mengzhong. The formula of centroid is derived by mathematical induction. [J].Journal of Jiujiang Teacher's College, 2002, 05: 46-4

[19] Liuzhiqin, The gesture recognition based on computer vision [D].Anhui University, 2014

[20] minutes

[21] kandyer. OpenGL Transform (EB/OL). http://blog.csdn.net/kandyer/article/details/12449973, 2016 01-18.

[22] School of Geodesy and Geomatics of Wuhan University. Error theory and measurement adjustment [M].Wuhan: wuhan university press, 2003.

\section{AUTHORS}

\section{Cai Mengmeng}

Master's degree of University of Jinan

The main research direction is Human-computer interaction and virtual reality.

E-mail: 1414663370@qq.com

\section{Zhiquan Feng}

Feng Zhiquan is a professor of School of Information Science and Engineering, University of Jinan. He got the Master degree from north-western polytechnical university, china in 1995, and Ph.D degree from Computer Science and Engineering Department, shandong university in 2006. He has published more than 50 papers on international journals, national journals, and conferences in recent years. His research interests include: human hand tracking/recognition/interaction, virtual reality, human-computer interaction and image processing
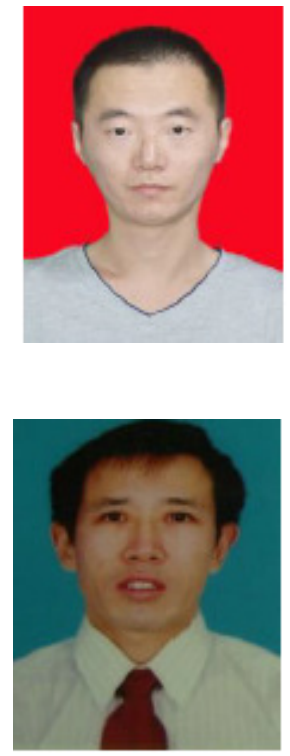
International Journal of VLSI design \& Communication Systems (VLSICS) Vol.8, No.1, February 2017

\section{Luan Min}

Master's degree of University of Jinan

The main research direction is Human-computer interaction and virtual reality.

E-mail: 1562920346@qq.com

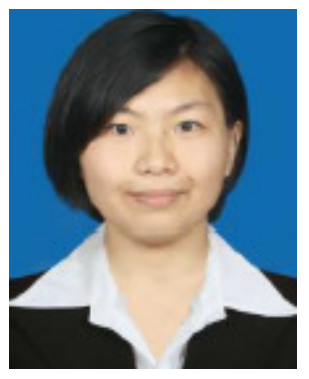

